\title{
Efeitos do exercício físico no controle da hipertensão arterial em idosos: uma revisão sistemática
}

\author{
Efeets of exerciseonhypetension cortrd indder adlts sysenticreview
}

\section{Resumo}

O exercício físico é uma das principais terapêuticas utilizadas para o paciente hipertenso, pois reduz a pressão arterial (PA) e os fatores de risco cardiovasculares, diminuindo a morbimortalidade. Objetivo: Analisar os efeitos do exercício físico na PA de idosos hipertensos, com base nos resultados de pesquisas empíricas realizadas no período de 2000 a 2010. Metodologia: Revisão sistemática de estudos experimentais, em inglês, português e espanhol, nas bases eletrônicas MEDLINE, PubMed, LILACS, Cochrane e PEDro, publicados entre 2000 e 2010, utilizando os descritores hipertensão, atividade física, exercício físico, idoso, exercício aeróbio e treinamento de resistência. Resultados: Foram encontrados 19 artigos e incluídos 12 artigos, sendo divididos em categorias temáticas: exercício aeróbico (6 artigos), exercício resistido (4), exercício aeróbico associado ao resistido (2). Entre os exercícios aeróbicos, três artigos evidenciaram redução na pressão arterial sistólica (PAS) e pressão arterial diastólica (PAD). Três artigos afirmam que treinamento resistido reduz significativamente os valores de PAS em repouso e pressão arterial média (PAM), apenas um artigo não registrou uma redução significativa na PAD e frequência cardíaca (FC) de repouso. A utilização dos exercícios aeróbicos associados aos resistidos foram superiores aos demais, pois apontaram reduções significativas na PAS, PAD, PAM e FC de repouso, confirmando as recomendações da VI Diretriz Brasileira de Hipertensão Arterial, mas os estudos em idosos são escassos. Conclusão: Esta revisão confirma os benefícios oriundos da prática do exercício físico na redução da PA após o exercício em idosos hipertensos.

1 Curso de Fisioterapia, Centro de Ciências da Saúde. Universidade de Fortaleza. Fortaleza, CE, Brasil.

2 Programa de Pós-Graduação em Saúde Coletiva. Centro de Ciências da Saúde. Universidade de Fortaleza. Fortaleza, CE, Brasil.

3 Programa de Bolsas de Iniciação Científica-PIBIC/CNPq, Universidade de Fortaleza. Fortaleza, CE, Brasil.

4 Curso de Fisioterapia, Faculdade Metropolitana de Fortaleza. Fortaleza, CE, Brasil.
Ingrid Correia Nogueira'

Zélia Maria de Sousa Araújo Santos² Daniela Gardano Bucharles Mont'Alverne ${ }^{3}$ Aline Barbosa Teixeira Martins ${ }^{4}$ Clarissa Bentes de Araujo Magalhães'

\section{Palavras-chave:}

Hipertensão. Idoso. Exercício físico. Exercício aeróbio.

Treinamento de resistência. 
Abstract

Physical exercise is one of the main techniques used for hypertensive patients because it reduces blood pressure (BP) and cardiovascular risk factors, reducing morbidity and mortality. Objective: To analyze the effect of exercise on BP in elderly hypertensive patients, based on the findings of empirical research conducted from 2000 to 2010. Methodology: A systematic review of experimental studies in English, Portuguese and Spanish, in the electronic databases MEDLINE, PubMed, LILACS, Cochrane, and PEDro, published between 2000 and 2010 using the keywords hypertension, physical activity, exercise and the elderly, aerobic exercise, resistance training. Results: We found 19 articles and selected 12, divided in thematic categories: aerobic exercise (6 articles) Resistance exercise (4), aerobic exercise associated with resistance (2). Among the aerobic exercise, three articles showed a reduction in SBP and DBP. Three articles argued that resistance training significantly reduces SBP and MBP at rest, and just one article reported a significant reduction in DBP and resting HR. The use of aerobic exercise associated with resistance was superior to others, as they showed significant reductions in SBP, DBP, MAP and HR at rest, confirming the recommendations of the Brazilian Guidelines on Hypertension, but studies in the elderly are scarce. Conclusion: This review confirms the benefits of exercise in reducing blood pressure after exercise in elderly hypertensive patients.
Key words: Hypertension. Elderly. Exercise. Aerobic exercise. Resistance training.

\section{INTRODUÇÃO}

O exercício físico (EF) é uma atividade física planejada, estruturada e repetitiva, que tem como objetivo final ou intermediário aumentar ou manter a saúde e a aptidão física, ${ }^{1}$ podendo propiciar benefícios agudos e crônicos. Dentre eles destacam-se a melhora no condicionamento físico; a diminuição da perda de massa óssea e muscular; o aumento da força, coordenação e equilíbrio; a redução da incapacidade funcional, da intensidade dos pensamentos negativos e das doenças físicas; e a promoção da melhoria do bem-estar e do humor, ${ }^{2}$ além da redução da pressão arterial (PA) pós-exercício em relação aos níveis pré-exercício. ${ }^{3,4}$

O efeito protetor do EF vai além da redução da $\mathrm{PA}$, estando associado à redução dos fatores de risco cardiovasculares e à menor morbimortalidade, quando comparadas pessoas ativas com indivíduos de menor aptidão física, o que explica a recomendação deste na prevenção primária e no tratamento da hipertensão.,

Nas últimas décadas, o EF tem sido incorporado como uma das principais terapêuticas do paciente hipertenso, associada ao tratamento medicamentoso e às modificações de hábitos alimentares e comportamentais. ${ }^{7}$
O EF deve ser avaliado e prescrito em termos de intensidade, frequência, duração, modo e progressão. A escolha do tipo de atividade física deverá ser orientada de acordo com as preferências individuais, respeitando as limitações impostas pela idade, como evitar o estresse ortopédico. ${ }^{8}$

Paralelamente ao envelhecimento, ocorre o aumento da inatividade física, entre os idosos, como demonstrado no último levantamento do Ministério da Saúde, fator de risco que contribui para o aumento da incidência de doenças crônicas, entre estas a hipertensão arterial. ${ }^{9-11}$ De fato, o idoso é mais suscetível aos efeitos adversos do sedentarismo, ao exercício físico de intensidade elevada e à terapia medicamentosa, sendo necessária maior compreensão dos efeitos do envelhecimento associados a esses fatores. ${ }^{12,13}$

Diante do exposto, surgiu a necessidade de investigar as características peculiares do EF (tipo, intensidade, duração) necessárias para promover uma queda pressórica significativa após sua execução. Entretanto, apesar dos grandes avanços no estudo dos efeitos agudos e crônicos do EF, existem algumas lacunas, principalmente em relação à sua aplicação na população idosa. 
Mediante o panorama apresentado, questionase: qual o tipo de exercício mais indicado para os idosos hipertensos? Qual a intensidade e a duração ideal para que ocorra a hipotensão pós-exercício nesta população? Diante destes questionamentos, optou-se pela realização de uma revisão sistemática, com o objetivo de analisar o efeito do exercício físico na pressão arterial de idosos hipertensos, com base nos achados de pesquisas empíricas realizadas durante o período de 2000 a 2010.

Os resultados deste estudo nortearão o planejamento de exercício físico para a clientela idosa, com vistas ao controle da PA, bem como à promoção da saúde em geral.

\section{MATERIAL E MÉTODO}

Trata-se de uma revisão sistemática, desenvolvida com artigos originais, publicados no período de 2000 a 2010. A escolha deste recorte temporal se deve aos inúmeros incentivos, inclusive pela mídia, no combate ao sedentarismo ocorrido nos últimos anos.

As bases eletrônicas consultadas foram: MEDLINE, PubMed, LILACS, Cochrane e PEDro. Para a busca e seleção dos artigos, utilizaram-se os procedimentos: palavras-chave, nas línguas português, inglês e espanhol hipertensão, (bypertension, hipertensión); atividade física, (physical activity, la actividad física); exercício físico (physical exercise, el ejercicio físico); idoso (elderly, anciano); exercício aeróbio (aerobic exercise, el ejercicio aeróbico); treinamento de resistência (resistance training, entrenamiento de resistencia). A busca se limitou aos artigos escritos em inglês, português e espanhol.

Os artigos identificados pela estratégia de busca inicial foram avaliados independentemente por dois autores, conforme os seguintes critérios de inclusão: (1) população (idosos); (2) intervenção (exercício físico); (3) desfecho (mensuração da pressão arterial antes e após o exercício físico); (4) tipo de estudo (experimentais). Aqueles aprovados pelos dois pesquisadores eram incluídos no estudo. Os que apresentassem discordância eram submetidos a um terceiro avaliador. Foram excluídos do estudo artigos com equívocos metodológicos e que não atendiam à proposta do estudo.

Foram encontrados 19 artigos nas bases de dados: dois na PubMed, quatro no LILACS, nove na MEDLINE, um na Cochrane e três no PEDro. Retiradas as referências cruzadas redundantes, constantes em mais de uma base, foram selecionados 14 artigos, mas incluídos somente 12. Dois artigos foram excluídos da revisão, devido aos seguintes motivos: o primeiro não possuía o ano de publicação da revista e o outro não descrevia adequadamente o protocolo utilizado.

Após a leitura dos artigos, com base nas categorias temáticas, as informações foram registradas em uma ficha catalográfica para cada trabalho, cujo roteiro conteve os dados: autor, ano, local do estudo, objetivo, tipo do estudo, amostra, técnica utilizada, parâmetros mensurados e resultados que compuseram as variáveis do estudo.

A organização dos dados dos artigos foi realizada, após as leituras - analítica e sintética. Foram selecionadas as seguintes categorias temáticas para facilitar a apresentação dos achados: exercício aeróbico, exercício resistido e exercício aeróbico associado ao resistido.

\section{RESULTADOS E DISCUSSÃO}

Para o processamento da análise, organizamos os resultados nas temáticas - exercício aeróbico, exercício resistido, exercícios aeróbicos associados aos de resistência.

\section{Exercício aeróbico}

De acordo com o quadro 1, Sanhueza \& Mascayano $^{14}$ realizaram pesquisa com o objetivo de analisar o impacto de um protocolo de exercícios sobre a PA de idosos hipertensos. Os participantes foram randomizados em dois grupos: grupo experimental (GE) e grupo 
controle (GC). O protocolo do GE foi composto por exercícios aeróbicos com intensidade de 70 a $80 \%$ do consumo máximo de oxigênio $\left(\mathrm{VO}_{2}\right.$ max), com frequência de três vezes por semana e duração de dez semanas. O GC realizou apenas a atividade física habitual.

Quadro 1 - Estudos realizados com o objetivo de analisar o efeito do exercício aeróbico sobre a PA de idosos hipertensos, no período de 2000 a 2010.

\begin{tabular}{|c|c|c|c|c|}
\hline $\begin{array}{c}\text { Autor(es)/ } \\
\text { Ano }\end{array}$ & Amostragem & Protocolo de exercício & $\begin{array}{l}\text { Parâmetros } \\
\text { mensurados }\end{array}$ & Resultados \\
\hline $\begin{array}{l}\text { HURCH, et al., } \\
2007\end{array}$ & $\begin{array}{l}427 \text { mulheres de meia } \\
\text { idade sedentárias. }\end{array}$ & $\begin{array}{l}4 \text { grupos, sendo um } \\
\text { controle, e os demais } \\
\text { experimentais, com base } \\
\text { no gasto energético pré- } \\
\text { estipulado } 4,8 \text { e } 12 \mathrm{Kcal} / \\
\mathrm{Kg} \text { com frequência } \\
\text { semanal de } 4 \text { vezes, } \\
\text { duração de } 6 \text { meses e, } \\
\text { intensidade baseada de } \\
50 \% \mathrm{VO}_{2} \text { max. }\end{array}$ & $\mathrm{VO}_{2}, \mathrm{PA}$ & $\begin{array}{l}\uparrow \mathrm{VO}_{2} \text { max nos grupos } \\
\text { experimentais, } \downarrow \text { PAS no } \\
\text { grupo com gasto energético } \\
\text { de } 12 \mathrm{Kcal} / \mathrm{Kg} \text {. }\end{array}$ \\
\hline $\begin{array}{l}\text { SANHUEZA, } \\
\text { MASCAYANO, } \\
2006\end{array}$ & $\begin{array}{l}37 \text { idosos randomizados } \\
\text { aleatoriamente em GE ( } \\
=18) \text { e } \mathrm{GC}(\mathrm{n}=19) \text {. }\end{array}$ & $\begin{array}{l}\text { Exercício aeróbico, } 3 \mathrm{x} \\
\text { por semana com duração } \\
\text { de } 10 \text { semanas com } \\
\text { intensidade de } 70-80 \% \\
\text { de } \mathrm{VO} 2 \text { max. O treino } \\
\text { consta de } 15 \mathrm{~min} \text { de } \\
\text { aquecimento, } 30 \mathrm{~min} \\
\text { de EA e, } 15 \text { min de } \\
\text { desaquecimento. }\end{array}$ & PAS, PAD e PAM & $\begin{array}{l}\text { O GE obteve uma } \downarrow \\
\text { significante nas variáveis de } \\
\text { PAM, PAS e PAD. }\end{array}$ \\
\hline $\begin{array}{l}\text { ZAGO et al., } \\
2010\end{array}$ & $\begin{array}{l}32 \text { idosas pré-hipertensas } \\
\text { dividas em } 2 \text { grupos } \\
\text { de acordo com o } \\
\text { polimorfismo T-786C } \\
\text { do gene } \\
\text { da e NOS }(\mathrm{TT}=20 \mathrm{~T}) \text { e } \\
(\mathrm{C}+\mathrm{CC}=12) .\end{array}$ & $\begin{array}{l}\text { Exercícios em esteira } \\
\text { ergométrica, durante } 6 \\
\text { meses, três vezes por } \\
\text { semana, com intensidade } \\
\text { progressiva durante as } \\
\text { semanas até atingir } 40 \\
\text { minutos e } 70 \% \mathrm{VO}_{2} \\
\text { máximo. }\end{array}$ & $\begin{array}{l}\mathrm{VO}_{2} \max , \mathrm{NO}, \\
\mathrm{FS}, \mathrm{RVP} \text { e PA }\end{array}$ & $\begin{array}{l}\uparrow \mathrm{VO}_{2} \text { max em ambos os } \\
\text { grupos. Não houve diferença } \\
\text { estatística entre os grupos } \\
\text { nas variáveis PAS, PAD, FS e } \\
\text { RVP. Aumento das relações } \\
\text { existentes entre NO, PA e } \\
\text { FS em idosas portadores do } \\
\text { alelo C. }\end{array}$ \\
\hline $\begin{array}{l}\text { KATHLEEN et } \\
\text { al., } 2009\end{array}$ & $\begin{array}{l}23 \text { indivíduos pré- } \\
\text { hipertensos divididos } \\
\text { em dipper }(\mathrm{n}=11) \text { e não } \\
\text { dipper }(\mathrm{n}=12) .\end{array}$ & $\begin{array}{l}\text { EF durante } 24 \text { semanas, } \\
3 x \text { por semana com } \\
\text { protocolo de } 50 \% \text { a } 70 \% \\
\text { de } \mathrm{VO}_{2} \text { max. }\end{array}$ & $\begin{array}{l}\text { PAS, PAD, } \\
\text { MAPAdip, } \\
\mathrm{VO}_{2} \text { max, NO, } \\
\mathrm{HDL}, \mathrm{LDL}, \\
\text { triglicerídeos e } \\
8 \text {-iso-PGF2 } \alpha\end{array}$ & $\begin{array}{l}\text { Não houve diferenças } \\
\text { significativas entre os } \\
\text { grupos em relação à média } \\
\text { de } 24 \mathrm{~h} \text { PAS, PAD, PAM, } \\
\text { LDL, HDL, triglicerídeos, } \\
\mathrm{VO}_{2} \text { max, } 8 \text {-iso-PGF2 } \alpha \text { e } \\
\text { NO. Ocorreram mudanças } \\
\text { estatisticamente significantes } \\
\text { entre as variáveis: MAPAdip, } \\
\text { descenso noturno e aos níveis } \\
\text { de colesterol. }\end{array}$ \\
\hline
\end{tabular}




\begin{tabular}{|c|c|c|c|c|}
\hline $\begin{array}{l}\text { WESTHOFF et } \\
\text { al., } 2007\end{array}$ & $\begin{array}{l}52 \text { idosos- } \\
\text { Com betabloqueador } \\
(\mathrm{n}=23) ; \text { Sem } \\
\text { betabloqueador }(\mathrm{n}=29) \\
\text { Foram randomizados } \\
\text { para GE } \\
(\mathrm{n}=25) \\
\mathrm{GC}(\mathrm{n}=27) .\end{array}$ & $\begin{array}{l}\text { Exercício na esteira. } \\
\text { Duração } 3 \text { meses, } 3 \\
\text { vezes por semana. Com } \\
\text { aumento gradativo do } \\
\text { tempo ( } 30 \text { até } 45 \mathrm{~min} \text { ) } \\
\text { e da intensidade do } \\
\text { exercício (até alcançar o } \\
\text { lactato } 2,0 \mathrm{mmol} / \mathrm{l}) \text {. }\end{array}$ & $\begin{array}{l}\text { PAS, PAD, PA aos } \\
\text { esforços, IMC, } \\
\text { lactato sanguíneo }\end{array}$ & $\begin{array}{l}\downarrow \text { significativa na PAS, PAD, } \\
\text { PA de esforço no GE. FC } \\
\text { média de treinamento foi } \\
\text { significativamente } \\
\text { menor nos pacientes em uso } \\
\text { de betabloqueadores. }\end{array}$ \\
\hline $\begin{array}{l}\text { MADDEN et } \\
\text { al., } 2009\end{array}$ & $\begin{array}{l}36 \text { idosos } \\
\text { Randomizados em dois } \\
\text { grupos: } \\
\text { EA }(n=16) ; \text { ER }(n=16) .\end{array}$ & $\begin{array}{l}\text { Duração de } 3 \text { meses, } 3 \\
\text { vezes por semana. } \\
\text { EA: intensidade } \\
\text { moderada a vigorosa } \\
\text { ( } 60 \text { a } 75 \% \text { da FC max) e } \\
\text { duração de } 60 \text { min (10min } \\
\text { de aquecimento, } 40 \\
\text { min de EA e } 10 \text { min de } \\
\text { resfriamento). } \\
\text { ER: Treinamento de força } \\
\text { com halteres e exercícios. }\end{array}$ & $\begin{array}{l}\text { FC, PAS, PAD, } \\
\text { PAM, } \\
\mathrm{VO}_{2} \text { max, } \\
\text { glicemia, perfil } \\
\text { lipídico, análise da } \\
\text { rigidez arterial. }\end{array}$ & $\begin{array}{l}\text { Não houve diferença } \\
\text { significativa entre os grupos } \\
\text { com relação à FC, PAS, PAD, } \\
\text { glicemia ou perfil lipídico. } \\
\text { O EA reduziu } \\
\text { significativamente a rigidez } \\
\text { arterial. }\end{array}$ \\
\hline
\end{tabular}

IMC: índice de massa corpórea; PA: pressão arterial; PAS: pressão arterial sistólica; PAD: pressão arterial diastólica; PAM: pressão arterial média; FC: frequência cardíaca; $\mathrm{VO}_{2}$ : consumo de oxigênio; GC: grupo controle; GE: grupo experimental; EF: exercício Físico; LDL: lipoproteínas de baixa densidade; HDL: lipoproteínas de alta densidade; 8-iso-PGF2 $\alpha$ : isoprostano 8; NO: óxido nítrico; EA: exercício aeróbico; ER: exercício resistido

Os autores ainda afirmam que o exercício aeróbico é uma ferramenta eficaz no tratamento de idosos hipertensos. Ao comparar os valores da mudança de pressão arterial média (PAM), pressão arterial sistólica (PAS) e pressão arterial diastólica (PAD) entre os grupos, o grupo experimental obteve uma diminuição estatisticamente significativa em todas as variáveis. ${ }^{14}$

O EF do tipo aeróbico corresponde aos processos metabólicos de produção de energia por meio do sistema oxidativo. Enfatizam-se os exercícios dinâmicos, repetitivos e submáximos de grandes grupos musculares. ${ }^{15,16}$

Durante os EFs aeróbicos, as contrações são seguidas de movimentos articulares, não existindo obstrução mecânica do fluxo sanguíneo. Há assim um aumento da atividade nervosa simpática, que por sua vez causará um incremento da frequência cardíaca (FC), do débito cardíaco (DC) e do volume sistólico e uma redução da resistência vascular periférica (RVP). Dessa forma, durante os exercícios dinâmicos observa-se aumento da PAS e manutenção ou redução da PAD. ${ }^{17}$
Os efeitos agudos tardios do EF são aqueles observados nas primeiras 24 ou 48 horas (ou até 72h) após o exercício, levando a uma discreta redução dos níveis tensionais. Já os crônicos adaptativos resultam da exposição regular, associando-se a adaptações fisiológicas que ocorrem num prazo mais longo, decorrentes de treinamento regular e dependentes do tipo de sobrecarga aplicada. Em indivíduos treinados, verifica-se atenuação da hipertensão arterial sistêmica (HAS), levando à bradicardia de repouso. ${ }^{18-20}$ Porém, para que a hipotensão pósexercício tenha importância clínica, é necessário que ela tenha magnitude importante e perdure por um período superior a 24 horas subsequentes à finalização do EF.

Aóxido nítrico sintase endotelial(eNOS) exerce grande influência no controle cardiovascular, o gene da eNOS consiste de 26 exons e 25 introns, localizados no braço longo do cromossomo 7 , na posição $7 \mathrm{q} 35$ a 36. Os principais polimorfismos do gene da eNOS encontrados na população são denominados de T-786C e G-894T. A substituição do nucleotídeo $\mathrm{T}$ (timina) pelo $\mathrm{C}$ (citosina) na posição 786 e/ou, do nucleotídeo G (guanina) 
pelo T (timina) na posição 894 no gene da eNOS pode refletir em alterações na atividade promoter deste gene, com redução na transcrição da eNOS e, consequentemente, diminuição na produção de NO. ${ }^{21}$ Sandrim $^{22}$ cita em seu estudo que o polimorfismo T-786C tem sido responsável por uma diminuição de 30 a 50\% da atividade promoter do gene da eNOS, ou seja, especificamente para o polimorfismo T-786C, os indivíduos portadores do alelo c (TC ou CC) possuem uma menor expressão do gene da eNOS com relação aos indivíduos não portadores do alelo c (TT). ${ }^{21}$

Zago et al. ${ }^{23}$ realizaram um estudo com mulheres idosas, pré-hipertensas, com o objetivo de investigar a influência do treinamento aeróbico e do polimorfismo T-786C, nas concentrações dos metabólitos do óxido nítrico (NOx), no fluxo sanguíneo (FS) e na PA. É importante ressaltar que o polimorfismo T-786C do gene da eNOS e a produção de ânion superóxido podem diminuir a produção e biodisponibilidade do óxido nítrico, comprometendo o grau de vasodilatação, efeito que pode ser revertido pelo exercício físico. Trinta e duas mulheres pré-hipertensas foram divididas em dois grupos, de acordo com o polimorfismo T-786C do gene (T'T - 20 participantes e TC+CC - 12 participantes).

No que se refere aos participantes do presente estudo, estes foram submetidos a um programa supervisionado de EFs aeróbicos, em esteira ergométrica, durante seis meses, três vezes por semana, com intensidade progressiva até atingir 40 minutos e $70 \% \mathrm{VO}_{2}$ máximo. Nenhuma diferença estatística foi encontrada entre os grupos nas variáveis PAS, PAD e índice de massa corpórea (IMC), fluxo sanguíneo (FS) e na RVP antes e após o programa de EF. $\mathrm{O} \mathrm{VO}_{2}$ máximo aumentou significativamente em ambos os grupos, após o programa de EF.

Rossi et al. ${ }^{24}$ encontraram um efeito positivo do programa de EF com relação ao polimorfismo T-786C do gene da eNOS. Isso sugere que um aumento do nível de EF regular pode melhorar a resposta ao controle cardiovascular, especialmente do NO, em portadores do alelo C, contribuindo para a relação entre PA e FS. O alelo $\mathrm{C}$ é considerado capaz de gerar efeitos deletérios no sistema cardiovascular em ambos os genótipos (TC e CC), e demonstram respostas similares, diferentemente do genótipo TT. ${ }^{24}$

O EF pode regular a vasodilatação mediada pelo $\mathrm{NO}$, diminuindo os valores de PA nos indivíduos hipertensos. ${ }^{25} \mathrm{O}$ EF regular possui a capacidade de diminuir a PA em aproximadamente $75 \%$ dos indivíduos hipertensos. Segundo os autores, o treinamento com intensidade moderada parece gerar maiores benefícios do que o de alta intensidade, para tais reduções na PA. ${ }^{26}$

O primeiro ensaio clínico randomizado que investiga os efeitos do treinamento cardiovascular sobre os níveis de lactato sanguíneo, PA e função vascular em idosos hipertensos que utilizam ou não betabloqueador foi realizado por Westhoff et al. ${ }^{27}$ Os participantes foram randomizados em dois grupos: GE (treinamento aeróbico realizado três vezes por semana durante 12 semanas, com incrementos no tempo de 30 a 45 minutos) e GC (não recebeu intervenção). A intensidade do treinamento do GE correspondia à velocidade necessária para alcançar a concentração de lactato sanguíneo de $2.0 \mathrm{~mol} / \mathrm{L}$. Deste modo, eram necessários incrementos na velocidade e no tempo de treinamento, visando a alcançar a concentração de ácido láctico desejada. A PA e a FC eram constantemente mensuradas durante o treinamento.

Os achados do estudo supracitados permitem afirmar que o programa de EF aeróbicos de 12 semanas favoreceu uma acentuada melhoria do desempenho físico, reduzindo significativamente a PA ao esforço, PAS e PAD em um curto período de tempo. É importante ressaltar que a diminuição da PA não pode ser atribuída a uma redução do peso corporal, pois não houve redução estatisticamente significante do IMC. Ao analisar os níveis de lactato sanguíneo, verificouse que o treinamento de resistência cardiovascular traz benefícios na presença ou ausência de betabloqueadores, incluindo acentuada melhoria da função endotelial. Porém, a FC de treinamento dos hipertensos que utilizam os betabloqueadores é cerca de 20\% menor quanto comparados aos que não utilizam o medicamento. 
Church et al. ${ }^{28}$ realizaram um ensaio clínico randomizado com 427 mulheres pós-menopausa, hipertensas e com sobrepeso ou obesas. Um dos principais objetivos era avaliar os níveis de exercício de 50\% abaixo e acima do gasto energético recomendado. As participantes foram randomizadas em quatro grupos, sendo um grupo controle e os três grupos experimentais com intensidade baseada em 50\% do $\mathrm{VO}_{2}$ máximo e FC de treinamento, com quatro sessões semanais, totalizando seis meses de treinamento. Todos os grupos iniciaram com gasto energético de $4-\mathrm{kcal} / \mathrm{kg}$ durante a primeira semana, apenas um grupo continuou com a mesma intensidade de treinamento, havendo incrementos de $4 \mathrm{kcal} /$ $\mathrm{kg}$ para um dos grupos (totalizando $8 \mathrm{kcal} / \mathrm{kg}$ ), e incrementos de $8 \mathrm{kcal} / \mathrm{kg}$ para o grupo de intensidade máxima (totalizando $12 \mathrm{Kcal} / \mathrm{Kg}$ ).

Foi possível concluir que nenhum dos grupos de EF teve mudanças significativas na PAS ePAD, em comparação com o GC. A única diferença entre os grupos foi encontrada comparando-se o grupo de 4-kcal/kg por semana com grupo de $12-\mathrm{kcal} / \mathrm{kg}$ por semana em relação a uma diminuição da PAS de 3,3 mmHg no grupo 12$\mathrm{kcal} / \mathrm{kg}$ por semana. Os dados do estudo são semelhantes às mudanças na PAS relatada em metanálises por Cornelissen e Fagard ${ }^{29}-3,0$ $\mathrm{mmHg}$ e por Whelton et al. ${ }^{30}-3,8 \mathrm{mmHg}$.

Há várias razões possíveis para que o treinamento físico não tenha induzido melhorias significativas na PA desta população. A amostra foi composta por mulheres de meia idade, com PA elevada, com sobrepeso ou obesas, apresentando alto risco para desenvolvimento de doenças cardiovasculares. A intensidade de treinamento pode ter sido muito baixa em um dos grupos e somente alta intensidade de treinamento gerou decréscimos significativos na PAS. Outro fator agravante foi que não houve perda de peso significativa associada com o treinamento físico, podendo anular os benefícios para a PA.

A literatura aponta que o treinamento aeróbio reduz $\mathrm{PA}$ em pacientes hipertensos..$^{29}$ No entanto, existem evidências limitadas e conflitantes em relação ao efeito do EF sobre o fenômeno de imersão. Sturgeon et al. ${ }^{31}$ investigaram o efeito do EF no declínio da PA durante o sono, conhecido como fenômeno de imersão, em idosos pré-hipertensos ou com HAS estágio 1. Os participantes foram subdivididos em grupos: dipper e não dipper. Foram classificadas como não dipper pessoas em quem o declínio da PA noturna é atenuada ou ausente, apresentando redução na PA inferior a 10\% durante o sono, e dipper quando se verifica redução normal de 10 a $20 \%$ da PA noturna em relação ao seu valor diurno.

O protocolo consistiu de seis meses de treinamento aeróbico, com frequência semanal de três vezes, iniciando com 20 minutos de EF por sessão a 50\% do $\mathrm{VO}_{2}$ máximo. Em seguida, houve incrementos de cinco minutos a cada semana até atingir 40 minutos de $\mathrm{EF}$ a $50 \%$ do $\mathrm{VO}_{2}$ máximo. Posteriormente, a intensidade do EF foi aumentada em $5 \%$ a cada semana, alcançando $70 \%$ do $\mathrm{VO}_{2}$ máximo. Após seis meses de treinamento aeróbico, não houve diferenças significativas entre dippers e não dippers em relação à média de $24 \mathrm{~h}$ da PAS, PAD e PAM. Concluíram em seu estudo que ao comparar o antes com o após a intervenção, ocorreram mudanças estatisticamente significantes na monitorização ambulatorial da pressão arterial (MAPA) entre os grupos.

Madden et al. ${ }^{32}$ realizaram um ensaio clínico comparando o efeito do EF aeróbico versus o treinamento resistido sobre a PA, FC, rigidez arterial,IMC, perfillipídico, $\mathrm{VO}_{2}$ máximoemidosos hipertensos. Os sujeitos foram randomizados em dois grupos: grupo aeróbico e grupo anaeróbico. O treinamento teve duração de três meses, sendo realizadas três sessões semanais. $\mathrm{O} E F$ aeróbico consistiu de atividades de intensidade moderada a vigorosa, baseado em 60 a 75\% da FC máxima, com duração de 60 minutos, sendo composto de dez minutos de aquecimento, 40 de treinamento aeróbico (esteira e cicloergômetro) e dez de resfriamento. No grupo anaeróbico foram realizados EF para ganho de força, porém os autores não descrevem a intensidade da carga, nem o tempo do treinamento. Devido a este fator, analisamos apenas o EF aeróbico.

Foi possível demonstrar, no estudo Madden et al., ${ }^{33}$ que com uma intervenção relativamente curta de treinamento aeróbico é possível reduzir a 
rigidez arterial independente da etiologia de base (seja a diabetes tipo 2, a HAS, a hipercolesterolemia ou até mesmo o envelhecimento), sendo, portanto, considerado um tratamento de primeira linha.

O fato de não ter ocorrido decréscimo da PA pode ser atribuído às diversas comorbidades que os indivíduos do estudo apresentavam, sendo constituído de um grupo de alto risco para rigidez arterial e os consequentes fatores de risco cardiovasculares associados a esta condição. Este achado indica que os efeitos benéficos de treinamento aeróbio sobre a vasculatura podem ocorrer independentemente da ocorrência dos outros benefícios já conhecidos pela literatura pertinente.

\section{Exercício Resistido}

Com base no quadro 2, estudo de Costa et al. ${ }^{34}$ investigou os efeitos subagudos de uma sessão de exercícios com pesos no comportamento da PA pós-esforço de idosas treinadas e não treinadas com diagnóstico de HAS. A amostra foi submetida aleatoriamente a uma sessão controle, na qual permaneceram sentadas em repouso, por 40 minutos, e uma sessão experimental, realizando sete exercícios com pesos executados em duas séries de 10-15 repetições máximas (RM). A PA foi verificada pelo método auscultatório após dez minutos de repouso no período pré-exercício, em ciclos de 15 minutos, durante uma hora e após o término da sessão.

Quadro 2 - Estudos realizados com o objetivo de analisar o efeito do exercício resistido sobre a PA de idosos hipertensos no período de 2000 a 2010.

\begin{tabular}{|c|c|c|c|c|}
\hline $\begin{array}{c}\text { Autor(es)/ } \\
\text { Ano }\end{array}$ & Amostragem & Protocolo de exercício & $\begin{array}{l}\text { Parâmetros } \\
\text { mensurados }\end{array}$ & Resultados \\
\hline $\begin{array}{l}\text { COSTA et } \\
\text { al., } 2010\end{array}$ & $\begin{array}{l}15 \text { mulheres idosas } \\
\text { realizaram os protocolos } \\
\text { por no mínimo } 20 \\
\text { semanas: } \\
\text { GT ( } \mathrm{n}=6 \text { ) treinamento } \\
\text { com peso, 3x por semana; } \\
\text { GNT ( } \mathrm{n}=9 \text { ) alongamentos. } \\
\text { 2x por semana. }\end{array}$ & $\begin{array}{l}2 \text { sessões: } \\
\text { sessão controle (SC), na qual } \\
\text { permaneceram sentadas em } \\
\text { repouso por } 40 \text { min; sessão } \\
\text { experimental (SE), realizando } \\
\text { sete exercícios com pesos } \\
\text { executados em duas séries de } \\
\text { 10-15 repetições máximas. }\end{array}$ & $\begin{array}{l}\text { IMC, PAS, PAD, } \\
\text { PAM }\end{array}$ & $\begin{array}{l}\text { Hipotensão pós-exercício } \\
\text { sendo mais consistentes nas } \\
\text { não treinadas. }\end{array}$ \\
\hline $\begin{array}{l}\text { JANNING } \\
\text { et al., } 2009\end{array}$ & $\begin{array}{l}8 \text { idosos com hipertensão } \\
\text { arterial controlada. }\end{array}$ & $\begin{array}{l}\text { Três séries de } 12 \text { repetições } \\
\text { durante } 7 \text { dias para: } \\
\text { Protocolo } 1 \text { (P1) três exercícios } \\
\text { para membros inferiores e } \\
\text { superiores } \\
\text { Protocolo } 2 \text { (P2) inverso do P1 } \\
\text { Protocolo } 3 \text { (P3) exercícos } \\
\text { alternados }\end{array}$ & PAS e PAD & $\begin{array}{l}\text { P3 foi superior aos demais } \\
\text { protocolos com diferenças } \\
\text { significativas em todas as } \\
\text { seis verificações da PAS } \\
\text { e nas de } 10,20,30 \text { e } 60 \\
\text { minutos da PAD. }\end{array}$ \\
\hline $\begin{array}{l}\text { KRINSKI } \\
\text { et al., } 2008\end{array}$ & $\begin{array}{l}24 \text { idosas com hipertensão } \\
\text { arterial. }\end{array}$ & $\begin{array}{l}1 \text { sessão de exercício resistido } \\
\text { com peso, constituída por } \\
8 \text { estações, realizadas em } 3 \\
\text { séries de } 12 \text { repetições com } \\
\text { intensidade de } 50 \% \text { de } 1 \text { RM. }\end{array}$ & IMC, DC, PA & $\begin{array}{l}\text { Redução da PAD } \\
\text { estatisticamente significativa } \\
\text { para a condição de repouso. }\end{array}$ \\
\hline $\begin{array}{l}\text { TERRA et } \\
\text { al., } 2008\end{array}$ & $\begin{array}{l}46 \text { idosas hipertensas } \\
\text { divididas em GTR }(n=20) \\
\text { e GC }(n=26) \text {. }\end{array}$ & $\begin{array}{l}\text { GTR- ER realizado em } 12 \\
\text { semanas, } 3 \text { vezes por semana, } \\
\text { consistindo de } 3 \text { séries de } 12 \text {, } \\
10 \text { e } 8 \text { repetições, em dias } \\
\text { alternados com intensidade de } \\
60-80 \% \text { de } 1 \mathrm{RM} \text {. }\end{array}$ & $\begin{array}{l}\text { PAS, PAD, PAM, } \\
\text { FC, DP }\end{array}$ & $\begin{array}{l}\text { TR reduziu } \\
\text { significativamente os } \\
\text { valores de PAS em } \\
\text { repouso, PAM e DP. Não } \\
\text { houve redução significativa } \\
\text { na PAD e FC de repouso. }\end{array}$ \\
\hline
\end{tabular}

GT: grupo treinadas; GNT: grupo não treinadas; SC: sessão controle; SE: sessão experimental; IMC: índice de massa corpórea; PAS: pressão arterial sistólica; PAD: pressão arterial diastólica; PAM: pressão arterial média; DC: densidade corporal; GTR: grupo de treinamento resistido; GC: grupo controle; ER: exercício resistido; RM: repetição máxima; FC: frequência cardíaca; DP: duplo produto; TR: treinamento resistido 
Os principais achados deste estudo indicaram declínio da PAS após o EF em ambos os grupos, porém, de maneira mais consistente no grupo não-treinamento. $\mathrm{O}$ grupo treinamento apresentou redução da PAS somente aos 30 minutos de recuperação, ao passo que no grupo de não-treinamento ocorreu um decréscimo dos 15 aos 60 minutos na monitoração da PAS pósexercício. Com relação à PAD e à PAM, houve redução significativa apenas no grupo nãotreinamento durante o período de recuperação, durante os 15 e 30 minutos na PAD e após 60 minutos na PAM. Os resultados evidenciam que uma sessão de EF com pesos é capaz de promover hipotensão pós-exercício em mulheres idosas e hipertensas, sendo mais consistente nas não treinadas. ${ }^{35}$

Os resultados encontrados na literatura podem ser explicados pelos ajustes cardiovasculares ao treinamento, como a redução da PA para uma mesma intensidade de EF. Essa diminuição da PA talvez esteja atrelada à melhora da eficiência no recrutamento das fibras musculares, que possivelmente proporcionaria menor demanda sanguínea para a musculatura ativa durante o exercício, refletindo nas respostas da PA pós-exercício. ${ }^{36} \mathrm{O}$ presente estudo corrobora os achados do estudo de Fisher et al., ${ }^{36}$ que demonstrou que uma única sessão de exercícios resistidos foi capaz de provocar leve resposta hipotensiva sistólica durante o período de recuperação em mulheres de meia-idade normotensas e hipertensas.

Krinski et al. ${ }^{37}$ realizaram um estudo que teve como objetivo avaliar os efeitos cardiovasculares agudos do EF resistido em idosas com hipertensão estágio I. A amostra foi submetida a uma sessão de EF resistido, constituída por oito estações, realizadas de três séries com 12 repetições, carga de 50\% 1 RM. Entre seus principais achados encontram-se um ganho significativo da PAS após a quinta estação, seguido de decréscimo não significativo no $10^{\circ}$ minuto após a sessão de exercícios. Em relação aos valores de PAD, estes evidenciam um aumento significativo após a quinta estação, seguido de redução significativa no $10^{\circ}$ minuto após a sessão de exercícios. Para a
FC, houve acréscimo significativo após a quinta estação, permanecendo elevado no $10^{\circ}$ minuto após a sessão de exercícios.

Frente a esta realidade, é possível identificar que apesar das modificações cardiovasculares agudas associadas ao Exercício Resistido (ER) a propensão positiva à hipotensão somente é verificada em relação à PAD. De acordo com Polito et al., ${ }^{38}$ durante a execução desse tipo de atividade, o valor da PA tende a aumentar rapidamente e pode atingir valores elevados, pela ativação dos quimiorreceptores por meio da fadiga periférica.

A pesquisa de Krinski et al. ${ }^{37}$ contraria os achados do estudo de Fisher et al., ${ }^{36}$ no qual se demonstrou que uma única sessão de exercícios resistidos foi capaz de provocar leve resposta hipotensiva sistólica durante o período de recuperação em mulheres de meia-idade normotensas e hipertensas.

Terra et al. $^{39}$ verificaram o efeito do treinamento resistido sobre a PA, FC e duplo produto (DP) em mulheres idosas com hipertensão controlada. O grupo treinamento resistido foi submetido a um protocolo de 12 semanas, frequência de três vezes por semana em dias alternados, consistindo de três séries de 12,10 e 8 repetições, de intensidade variável em diferentes estágios do programa. Houve incremento progressivo da carga de treinamento a cada quatro semanas, iniciando com a carga de $60 \%$ de 1 RM, com incrementos de $70 \%$ de 1 RM e de $80 \%$ de 1 RM nas últimas semanas. Os exercícios foram realizados de forma alternada.

Após 12 semanas de treinamento resistido, ocorreram reduções significativas nos valores de PAS em repouso, PAM e DP em idosos com hipertensão controlada. Não foram encontradas reduções significativas na PAD e FC de repouso no grupo de treinamento e no controle, corroborando o estudo de Taaffe. ${ }^{40}$

Deve-se ressaltar que este foi o único trabalho que verificou a relação entre o DP com treinamento de resistência. A redução do DP em repouso desempenha papel significativo na 
redução dos riscos de problemas cardiovasculares. Assim, o treinamento de resistência pode levar a uma redução do trabalho cardiovascular, ajudando a reduzir o risco de infarto agudo do miocárdio e doenças coronarianas. Diante deste fato, o treinamento resistido pode ser usado como uma terapia não-medicamentosa, tanto para prevenção quanto para tratamento e controle da HAS.

O estudo de Janning et al. ${ }^{41}$ analisou a influência da ordem de execução dos EFs resistidos na hipotensão pós-exercício em idosos com HAS. A amostra foi composta por oito idosos que se submeteram aleatoriamente a três diferentes protocolos. No protocolo 1 (P1), foram realizados três EFs para membros inferiores (MI) seguidos de três EFs para membros superiores (MS). No protocolo 2 (P2), a situação foi inversa. Já o protocolo 3 (P3) foi organizado de maneira a intercalar um exercício para MS com um EF para MI.

Observou-se que o P1 não apresentou diferenças significativas em relação ao repouso quanto à PAS e PAD em nenhuma das seis verificações de PA pós-exercício. Dessa maneira, não ocorreu hipotensão pós-exercício no P1. Já no P2, ocorreram diferenças nas verificações de 20 e 40 minutos de recuperação da PAS, ocorrendo, assim, leve hipotensão pós-exercício. Porém, não apresentaram diferenças significativas em relação ao repouso quanto à PAD em nenhum momento da recuperação, portanto, não ocorreu hipotensão diastólica pós-exercício. No P3, foram encontradas diferenças em todas as verificações após a realização da sessão de exercícios resistidos. Assim, o P3 demonstrouse extremamente eficaz em produzir hipotensão pós-exercício, tanto da PAS quanto da PAD.

O treinamento contrarresistência é considerado relativamente seguro para aumentar a força muscular e melhorar a qualidade de vida, tanto em adultos saudáveis, quanto em idosos ou em portadores de comprometimentos cardiovasculares. ${ }^{38,42}$
Durante o EF de força, tanto a PAS quanto a PAD tendem a elevar, ocasionando aumento também expressivo na PAM, mesmo que por um período curto de tempo. Isso pode ser explicado pelas variáveis que concorrem para a elevação da PA e que se manifestam durante a atividade física de elevada intensidade, como a ativação de quimiorreceptores por fadiga periférica. Assim, exercícios realizados até a exaustão repercutiriam em uma resposta mais elevada da PA imediatamente após o esforço, diferentemente dos exercícios realizados de forma submáxima. ${ }^{42,43}$

Os valores de PA nos momentos subsequentes ao EF, contudo, parecem declinar de forma rápida, pelo mecanismo barorreflexo, pela hiperemia decorrente da contração muscular e pela supressão da atividade simpática. ${ }^{44,45}$ Além disso, os valores de PA podem se reduzir além daqueles observados na condição pré-exercício. Os mecanismos envolvidos nesse processo ainda estão pouco esclarecidos. É possível que diferentes vias fisiológicas, isoladas ou combinadas, contribuam para tal fenômeno, tais como maior liberação de óxido nítrico e menor descarga adrenérgica. Essa redução da PA após o EF é tida como uma das principais intervenções não-farmacológicas de controle da PA, principalmente em indivíduos hipertensos. ${ }^{46,47}$

Segundo Lizardo \& Simões, ${ }^{48}$ diferentes formas de EF resistidos resultam em hipotensão pós-exercício, mas as sessões envolvendo maior massa muscular, como os membros inferiores, apresentam efeito hipotensor mais significativo e duradouro em relação aos EFs que utilizam menor massa muscular, como os membros superiores, mesmo sendo realizados na mesma intensidade.

É importante ressaltar que, além dos cuidados quanto à intensidade, duração e frequência do treinamento, os idosos hipertensos devem receber instruções durante a realização dos EFs, com o objetivo de inibir a manobra de valsalva, que é um dos fatores que mais contribui para o aumento do risco cardiovascular durante exercícios com pesos. ${ }^{49}$ 


\section{Exercícios aeróbicos associados aos de resistência}

Conforme o quadro 3, Krinski et al. ${ }^{37}$ analisaram os efeitos do EF aeróbio e resistido no perfil antropométrico e respostas cardiovasculares de idosos portadores de HAS. $O$ protocolo de treinamento teve frequência semanal de três vezes e duração de seis meses. Foi constituído inicialmente de 20 minutos de EF aeróbio, desenvolvido em esteira, com intensidade de 60 a 70\% da FC máxima determinada e com o auxílio da escala de Borg sobre percepção subjetiva de esforço.

Quadro 3 - Estudos realizados com o objetivo de analisar o efeito do exercício aeróbico associado ao resistido sobre a PA de idosos hipertensos, no período de 2000 a 2010.

\begin{tabular}{|c|c|c|c|c|}
\hline $\begin{array}{c}\text { Autor(es)/ } \\
\text { Ano }\end{array}$ & Amostragem & Protocolo de exercício & $\begin{array}{l}\text { Parâmetros } \\
\text { mensurados }\end{array}$ & Resultados \\
\hline $\begin{array}{l}\text { KRINSKI, } \\
\text { et al., } 2006\end{array}$ & $\begin{array}{l}53 \text { idosos hipertensos } \\
\text { sedentários com } \\
\text { hipertensão estágio } 1 .\end{array}$ & $\begin{array}{l}20 \text { min de exercício aeróbio } \\
\text { e } 40 \text { min de exercícios } \\
\text { resistidos dinâmicos, com } \\
\text { frequência semanal de três } \\
\text { vezes e duração de seis meses. }\end{array}$ & $\begin{array}{l}\text { PA, FC, peso, IMC, } \\
\text { Percentual de gordura } \\
\text { corporal (GC\%). }\end{array}$ & $\begin{array}{l}\text { Redução linear na pressão } \\
\text { arterial média PAM, FC, } \\
\text { IMC, GC\%. }\end{array}$ \\
\hline $\begin{array}{l}\text { STEWART, } \\
\text { et al., } 2005\end{array}$ & $\begin{array}{l}104 \text { hipertensos } \\
\text { (idade }=55 \text { a } 75 \text { anos), } \\
\text { randomizados: } \\
\text { 53- GC } \\
\text { 51- GE }\end{array}$ & $\begin{array}{l}\text { GC: Orientações (dieta) e } \\
\text { atividade Física } \\
\text { GE: Treinamento aeróbico e } \\
\text { de resistência por } 26 \text { semanas, } \\
\text { com frequência semanal } \\
\text { de } 3 \text { vezes. Intensidade do } \\
\text { treinamento de resistência } \\
50 \% \text { de } 1 \text { RM, e do aeróbico } \\
60 \text { a } 90 \% \text { da FC máxima. }\end{array}$ & $\begin{array}{l}\text { PA, pico de oxigênio, } \\
\text { IMC, FC, resistência } \\
\text { arterial. }\end{array}$ & $\begin{array}{l}\downarrow \text { PAS, PAD, IMC. O GE } \\
\text { obteve } \uparrow \text { doVO }, \text { uma } \downarrow \text { do } \\
\text { IMC e da PAD média } \\
\text { quanto comparado ao } \\
\text { controles } \\
\downarrow \text { PAS não foi } \\
\text { significativamente } \\
\text { diferente entre os grupos. }\end{array}$ \\
\hline
\end{tabular}

PA: pressão arterial; FC: frequência cardíaca; IMC: índice de massa corpórea; GC\%: percentual de gordura corporal; PAM: pressão arterial média; GC: grupo controle; GE: grupo experimental; PAS: pressão arterial sistólica; PAD: pressão arterial diastólica; $\mathrm{VO}_{2}$ : consumo de oxigênio; RM: repetição máxima.

A segunda etapa foi baseada em 40 minutos de EF resistidos dinâmicos, executados de forma aleatória por meio do treinamento em circuito. Desenvolvido de forma concêntrica e excêntrica para membros superiores, tronco e membros inferiores, constituído de oito estações, cada uma composta por três séries completas de dez repetições cada, realizadas em ritmo moderado e contínuo com intensidade estimada em $60 \%$ de $1 \mathrm{RM}$, totalizando 60 minutos. Os ajustes das cargas do treinamento resistido foram realizados por meio de aplicação do teste de 1 RM, executado a cada dois meses.
Após seis meses de treinamento, os autores constataram que a utilização de um programa de treinamento físico baseado em exercícios aeróbios associados a exercícios de resistência (circuito com pesos), resultou em reduções significativas na PAM e FC de repouso, sendo acompanhados de uma redução linear no percentual de gordura corporal (\%GC) de idosos hipertensos. É importante salientar que, após os dois primeiros meses, estes valores foram menos evidentes em relação aos meses subsequentes de exercícios, tornando o ajuste das cargas de treinamento importantes no resultado final. 
Segundo Brum et al., ${ }^{18}$ o efeito hipotensor encontrado pode ser justificado pela redução do DC decorrente da diminuição da FC associada à melhora da resposta vasodilatadora. Para esses autores, os efeitos são ocasionados por uma maior disponibilidade de óxido nítrico, associados a uma diminuição da atividade nervosa simpática.

Stewart et al. ${ }^{50}$ realizaram um ensaio clínico randomizado, controlado, comparando o efeito do treinamento aeróbico com resistência combinada versus o aconselhamento sobre os cuidados habituais de atividade e dieta em pacientes hipertensos. Participaram do presente estudo indivíduos com HAS leve ou pré-hipertensos com idade entre 55 e 75 anos e que não necessitassem de medicação antihipertensiva, sendo necessário que a PA estivesse nos valores aceitáveis (PAS de 130 a $159 \mathrm{mmHg}$ e PAD de 85-99 $\mathrm{mmHg}$ ).

O estudo teve duração de seis meses. O GE foi submetido a 78 sessões, três dias por semana, durante 26 semanas. Cada sessão teve início com um alongamento, seguido por treinamento de resistência e aeróbio. O treinamento de resistência consistiu de duas séries de 10 a 15 repetições por exercício, utilizando uma carga de $50 \%$ de $1 \mathrm{RM}$. Os exercícios foram realizados de forma concêntrica e excêntrica para membros superiores, tronco e membros inferiores. Quando o participante completou 15 repetições de um exercício com facilidade, o peso foi aumentado. O EF aeróbio teve duração de 45 minutos, com intensidade de $60 \%$ a $90 \%$ da FC máxima e os participantes foram autorizados a escolher uma esteira, bicicleta estacionária, ou stairstepper (escada deslizante) para o seu treino.

Declínios nas PAS e PAD ocorreram em ambos os grupos, com maior redução na PAD, ocorrendo no GE. A ausência de melhora na rigidez aórtica sugere que pessoas idosas podem ser resistentes a induzidas melhorias na PAS pelo exercício. Apesar de reduções modestas no peso corporal e IMC, houve reduções notáveis, em geral na circunferência abdominal e aumento da massa corporal magra. Estas melhorias na composição corporal estão correlacionadas com a redução da PA. Esses achados sugerem que as mudanças na composição corporal, associadas ao treinamento físico, apresentam correlação significativa com a saúde cardiovascular em idosos. ${ }^{51}$

Ensaios clínicos controlados demonstraram que os EFs aeróbios, que devem ser complementados pelos resistidos, pois promovem reduções da PA, devem ser indicados para a prevenção e o tratamento da HAS, além de promover ações de promoção da saúde que melhoram a qualidade de vida do indivíduo..$^{30,52}$

Diante deste contexto, torna-se necessário manter uma boa saúde cardiovascular por meio de exercícios, pelo menos cinco vezes por semana, 30 minutos de EF moderado de forma contínua ou acumulada, desde que em condições de realizálo. A FC de pico deve ser avaliada pelo teste ergométrico, sempre que possível, e na vigência da medicação cardiovascular de uso constante. ${ }^{7}$

De acordo com a II Diretrizes da Sociedade Brasileira de Cardiologia (DBBC) $)^{52}$ sobre teste ergométrico, a recomendação é que, inicialmente, os indivíduos realizem atividades leves a moderadas. Somente após estarem adaptados, caso julguem confortável e não haja nenhuma contraindicação, é que devem passar às vigorosas. ${ }^{51,53,54}$

Sugestões da VI Diretrizes Brasileiras de Hipertensão Arterial para a intensidade de EFs isotônicos, segundo a frequência cardíaca: atividades leves - mantém-se com até $70 \%$ da FC máxima ou de pico, recomendando-se a faixa entre $60 \%$ e $70 \%$, quando se objetiva o treinamento efetivo eminentemente aeróbio; atividades moderadas - mantém-se entre $70 \%$ e $80 \%$ da FC máxima ou de pico, sendo considerada a faixa ideal para o treinamento que visa a prevenção e o tratamento da HAS; atividades vigorosas - mantém-se acima de $80 \%$ da FC máxima ou de pico, propondo-se a faixa entre $80 \%$ e $90 \%$ quando se objetiva o treinamento com expressivo componente aeróbio, desenvolvido já com considerável participação do metabolismo anaeróbio. 
Em relação aos EFs resistidos, recomenda-se que sejam realizados entre duas e três vezes por semana, por meio de uma a três séries de oito a 15 repetições, conduzidas até a fadiga moderada. É importante ressaltar que se recomenda a avaliação médica antes do início de um programa de treinamento estruturado e sua interrupção na presença de sintomas. Em hipertensos, a sessão de treinamento não deve ser iniciada se as PAS e PAD estiverem superiores a 160 e/ou $105 \mathrm{mmHg}$, respectivamente.

\section{CONCLUSÃO}

Com base nos achados da presente revisão, é possível identificar que o Exercício Físico aeróbico é uma ferramenta eficaz no tratamento da população de hipertensos idosos. O treinamento supervisionado, com frequência semanal de três vezes por semana, e com intensidade moderada parece gerar mais benefícios do que os de alta intensidade, para tais reduções na Pressão Arterial.
Em relação à combinação dos Exercícios Físicos (EF), foi possível constatar que a utilização de um programa de treinamento físico baseado em EF aeróbios associados a EF de resistência (circuito com pesos) resultou em reduções significativas na Pressão Arterial Média e Frequência Cardiáca de repouso, sendo superior à realização das modalidades de exercício de forma isolada, corroborando as recomendações da VI Diretrizes Brasileiras de Hipertensão Arterial. ${ }^{7}$

Existem, no entanto, alguns fatores que prejudicam a qualidade metodológica das pesquisas, em relação às características da população estudada, incluindo diferentes estágios de Hipertensão Arterial Sistêmica e duração da doença, patologias concomitantes e tratamentos com drogas diferentes para favorecer o número de participantes, reduzindo, assim, a validade interna dos estudos.

\section{REFERÊNCIAS}

1. Moraes H, Deslandes A, Ferreira C, Pompeu FAMS, Ribeiro P, Laks P. O exercício físico no tratamento da depressão em idosos: revisão sistemática. Rev. Psiquiatr, 2007; 29(1).

2. Fountoulakis KN, O’ Hara R, Iacovides A, Camilleri CP, Kaprinis S, Kaprinis G. Unipolar late-onset depression: a comprehensive review. Ann. Gen. Hosp. Psychiatry., 2003;2: 1-14.

3. Pescatelo LS, Franklin BA, Fagard R, Farquhar WB, Kelly GA, Ray CA. American College of Sports Medicine Position Stand. Exercise and hypertension. Med. Sci. Sports Exerc. 2004;36(3): 533-53.

4. Halliwill JR. Mechanisms and clinical implications of post-exercise hypotension in humans. Exerc. Sports. Sci. Rev., 2001; 29(2): 65-70.

5. Fagard, RH. Exercise is good for your blood pressure: effects of endurance training and resistance training. Clin. Exp. Pharmacol. Physiol., 2006; 36(9): 853-6.

6. Myers J, Prakash M, Froelicher V, Do D, Partington $\mathrm{S}$, Atwood JE. Exercise capacity and mortality among men referred for exercise testing. N. Engl. J. Med., 2002; 346: 793-801.
7. Sociedade Brasileira de Cardiologia. VI Diretrizes Brasileiras de Hipertensão Arterial. Rev Bras Hipertens; 2010; 17(1): 1-64.

8. Fletcher GF, Balady GJ, Amsterdam EA, Chaitman B, Eckel R, Fleg J. Exercise standards for testing and training: a statement for healthcare professionals from the American Heart Association. Circulation., 2001;104(14): 1694-1740.

9. BRASIL. Ministério da Saúde. Vigitel Brasil 2009: vigilância de fatores de risco e proteção para doenças crônicas por inquérito telefônico / Ministério da Saúde, Secretaria de Vigilância em Saúde, Secretaria de Gestão Estratégica e Participativa. Brasília: Ministério da Saúde, 2009.

10. Fagard RH; Cornelissen VA. Effect of exercise on blood pressure control in hypertensive patients. Eur. J. Cardiovasc. Prev. Rehabil., 2007;14(1): 12-17.

11. Baumam A. Updating the evidence that physical activity is good for health: an epidemiological review 2000-2003. J. Sci. Med. Sport., 2004; 7(1): 6-19.

12. Taylor-Tolbert NS, Dengel DR, Brown MD, Mc Cole SD, Pratley RE, Ferrel RE. Ambulatory blood pressure after acute exercise in older men with 
essential hypertension. Am. J. Hypertens., 2000; 13(1): 44-51.

13. Scher LML; Nobre F; Lima NKC. O papel do exercício físico na pressão arterial em idosos. Rev. Bras. Hipertens., 2008 ; 15(4): 228-231.

14. Sanhueza S; Mascayano M. Impacto del Ejercicio en el Adulto Mayor Hipertenso. Revista HCUC, 2006; 17(2): $111-128$.

15. Zilio, A. Treinamento físico: Terminologia. $2 \mathrm{ed}$. Canoas: Ulbra, 2005.

16. Kisner C; Colby LA. Exercícios terapêuticos: Fundamentos e Técnicas. $4^{\circ}$ ed. Barueri: Manole; 2005.

17. Forjaz CLM; Tinucci T. A medida da pressão arterial no exercício. Revista Brasileira de Hipertensão, 2000;7(1): 79-87.

18. Brum PC, Forjaz CLM, Tinucci T, Negrão CE. Adaptações agudas e crônicas do exercício físico no sistema cardiovascular. Rev. Paul. Educ. Fis., 2004; 18(2): 21-31.

19. Araújo CGS. Fisiologia do exercício e hipertensão arterial: breve introdução. Hipertensão, 2001; 14(3): 78-83.

20. Thompson PD, Crouse SF, Goodpaster B, Kelley D, Moyna N, Pescatello L. The acute versus chronic response to exercise. Med. Sci. Sports Exerc., 2001; 33(6): 438-435.

21. Zago AS; Kokubun E; Brown MD. Exercício físico como estímulo para o aumento da produção e biodisponibilidade do oxido nítrico e seu efeito no controle da pressão arterial. Arq. Ciênc. Saúde Unipar, 2009; 13(1): 59-66.

22. Sandrim VC. Influence of T-786C polymorphism on the promoter activity of eNOS. Clin Chim Acta., 2006; 367(1-2): 208.

23. Zago AS, Kokubun E, Stewart - Fenty N, Park JY, Attipoe S, Hagberg J. Efeito do Exercício Físico e do Polimorfismo T-786C na Pressão Arterial e no Fluxo Sanguíneo de Idosas. Arq. Bras. Cardiol., 2010; 95(4): 510-517.

24. Rossi GP, et al. The T-786C and Glu298Asp polymorphisms of the endothelial nitric oxide gene affect the forearm blood flow responses of Caucasian hypertensive patients. J. Am. Coll. Cardiol., 2003; 41(6): 938-45.

25. Rush JW; Denniss SG; Graham DA. Vascular nitric oxide and oxidative stress: determinants of endothelial adaptations to cardiovascular disease and to physical activity. Can J Appl Physiol., 2005; 30(4): 442-74.
26. Hagberg JM; Park JJ; Brown MD. The role of exercise training in the treatment of hypertension: an update. Sports Med., 2000; 30(3): 193-206.

27. Westhoff TH, Franke N, Schimidt S, Vallbracht Israng K, Zidek W, Dimeo F,et al. Beta-blockers do not impair the cardiovascular benefits of endurance training in hypertensives. Journal of Human Hypertension, 2007; 21(6): $486-493$.

28. Church TS, Earnest CP, Skinner JS, Blair SN. Effects of Different Doses of Physical Activity on Cardiorespiratory Fitness Among Sedentary, Overweight or Obese Postmenopausal Women with Elevated Blood Pressure a Randomized Controlled Trial. JAMA, 2007; 297(19): 2081 - 2091.

29. Cornelissen VA; Fagard RH. Effect of resistance training on resting blood pressure: a meta-analysis of randomized controlled trials. J. Hypertens.,2005; 23: 251-9.

30. Whelton SP, Chin A, Xin X, He J. Effect of aerobic exercise on blood pressure: a meta-analysis of randomized, controlled trials. Ann. Intern Med., 2002; 136(7): 493-503

31. Sturgeon KM, Stewart - Fenty NM, Diaz KM, Brinkley TE, Dowling TC, Brown MD. The relationship of oxidative stress and cholesterol with dipping status before and after aerobic exercise training. Blood Press, 2009 September; 18(4): 171-179.

32. Madden KM, Lockhart C, Cuff D, Potter TF, Meneilly GS. Short-Term Aerobic Exercise Reduces Arterial Stiffness in Older Adults With Type 2 Diabetes, Hypertension, and Hypercholesterolemia. Diabetes Care, 2009; 32(8): 1531-5.

33. Dart AM, Gatzka CD, Cameron JD, Kingwell BA, Liang Yu Lu, Berry KL. Large artery stiffness is not related to plasma cholesterol in older subjects with hypertension. Arterioscler. Thromb. Vasc. Biol., 2004; 24: 962-8.

34. Costa JBY, Gerage AM, Gonçalves CGS, Pina FLC, Polito MD. Influência do Estado de Treinamento Sobre o Comportamento da PressãoArterial Após uma Sessão de Exercícioscom Pesos em Idosas Hipertensas. Rev. Bras. Med. Esporte, 2010; 16(2): 103-6.

35. Parente V, D’Antona G, Adami R, Miotti D, Capodaglio P, De Vito G,et al. Long-term resistance training improves force and unloaded shortening velocity of single muscle fibers of elderly women. Eur. J. Appl. Physiol., 2008; 104(5): 885-893.

36. Fisher MM. The effect of resistance exercise on recovery blood pressure in normotensive and borderline hypertensive women. J. Strength. Cond. Res., 2001; 15: 210-216. 
37. Krinsk K, Elsangedy HM, Nardo Junior N, Soares IA. Efeito do exercício aeróbio e resistido no perfil antropométrico e respostas cardiovasculares de idosos portadores de hipertensão. Maringá, 2006; 28(1): 71-5.

38. Polito MD, Simão R, Senna GW, Farinatti PTV. Efeito hipotensivo do exercicio de forca realizados em intensidades diferentes e o mesmo volume de trabalho. Rev. Bras. Med. Esporte, 2003; 9(2): 69-73.

39. Terra DF, Mota MR, Rabelo HT, Bezerra LMA, Lima RM, Ribeiro AG, et al. Reduction of Arterial Pressure and Double Product at Rest after Resistance Exercise Training in Elderly Hypertensive Women. Arq. Bras. Cardiol., 2008; 91(5): 274-9.

40. Taaffe DR, Galvão DA, Sharman JE, Coombes JS. Reduced central blood pressure in older adults following progressive resistance training. J. Hum. Hypertens., 2007; 21(1): 96-8.

41. Jannig PR,Cardoso AC,Fleischmann E, Coelho CW. Carvalho T. Influência da Ordem de Execução de Exercícios Resistidos na Hipotensão Pos-exercicio em Idosos Hipertensos. Rev. Bras. Med. Esporte, 2009; 15(5): 338 - 341.

42. Adams K, et al. Progression models in resistance training for healthy adults. Med. Sci. Sports. Exerc., 2005; 4(2): 364-80.

43. Mediano MFF, Paravidino V, Simão R, Pontes FL, Polito MD. Comportamento subagudo da pressão arterial após o treinamento de força em hipertensos controlados. Rev. Bras. Med. Esporte., 2005; 11(6): $337-40$.

44. Carrington CA; White MJ. Exercise-induced muscle chemoreflex modulation of spontaneous baroreflex sensitivity in man. J. Physiol., 2001; 536(3):957-62.

45. Osada T, Katsumura T, Murase N, Sako T, Higuchi $\mathrm{H}$, Kiome R, et al. Post-exercise hyperemia after ischemic and non-ischemic isometric handgrip exercise. J. Physiol. Anthropol. Appl. Human Sci., 2003;22(6): 299-309.
46. Macdonald JR; Macdougall JD; Hogben CD. The effects of exercising muscle mass on post exercise hypotension. J Hum Hypertens., 2000; 14(5): 3172000 .

47. Halliwill JR; Dinenno, FA; Dietz NM. Alphaadrenergic vascular responsiveness during post exercise hypotension in humans. J. Physiol., 2003; 550(1): 279-86.

48. Lizardo JHF; Simões HG. Efeitos de diferentes sessões de exercícios resistidos sobre a hipotensão pós-exercício. Rev. Bras. Fisioter., 2005; 9(3): 289-295.

49. Polito MD; Farinatti PTV. Respostas de frequência cardíaca, pressão arterial e duplo-produto ao exercício contra-resistência: uma revisão da literatura. Revista Portuguesa de Ciências do Desporto, 2003;3(1): 79 - 91.

50. Stewart KJ. Effect of Exercise on Blood Pressure in Older Persons - A Randomized Controlled Trial. Arch Intern Med, 2005;165: 756-762

51. Fletcher GF, et al. Exercise standards for testing and training: a statement for healthcare professionals from the American Heart Association. Circulation. 2001; 104(14):1694-740.

52. Sociedade Brasileira de Cardiologia. II Diretriz de Reabilitação Cardiopulmonar e Metabólica: aspectos práticos e responsabilidades. Arq. Bras. Cardiol., 2006; 86(1).

53. Camarda SR, et al. Comparison of maximal heart rate using the prediction equations proposed by Karvonen and Tanaka. Arq Bras Cardiol. 2008; 91(5):311-4.

54. Merz CNB, et al. ACCF/AHA/ACP 2009 Competence and Training Statement: A Curriculum on Prevention of Cardiovascular Disease. A Report of the American College of Cardiology Foundation/ American Heart Association/American College of Physicians Task Force on Competence and Training (Writing Committee to Develop a Competence and Training Statement on Prevention of Cardiovascular Disease). Circulation, 2009; 120: 100-126. 